\title{
Polarized heat current generated by quantum pumping in two-dimensional topological insulators
}

\author{
F. Ronetti, ${ }^{1,2,3}$ M. Carrega, ${ }^{4}$ D. Ferraro, ${ }^{3}$ J. Rech, ${ }^{3}$ T. Jonckheere, ${ }^{3}$ T. Martin, ${ }^{3}$ and M. Sassetti ${ }^{1,2}$ \\ ${ }^{1}$ Dipartimento di Fisica, Università di Genova, Via Dodecaneso 33, I-16146 Genova, Italy \\ ${ }^{2}$ CNR-SPIN, Via Dodecaneso 33, I-16146 Genova, Italy \\ ${ }^{3}$ CNRS, CPT, Aix Marseille Université, Université de Toulon, Marseille, France \\ ${ }^{4}$ NEST, Istituto Nanoscienze-CNR and Scuola Normale Superiore, I-56127 Pisa, Italy \\ (Received 24 November 2016; revised manuscript received 6 February 2017; published 8 March 2017)
}

\begin{abstract}
We consider the transport properties of a two-dimensional topological insulator in a double quantum point contact geometry in the presence of a time-dependent external field. In the proposed setup an external gate is placed above a single constriction and it couples only with electrons belonging to the top edge. This asymmetric configuration and the presence of an ac signal allow for a quantum pumping mechanism, which, in turn, can generate finite heat and charge currents in an unbiased device configuration. A microscopic model for coupling with the external time-dependent gate potential is developed and the induced finite heat and charge currents are investigated. We demonstrate that in the noninteracting case, heat flow is associated with a single spin component, due to the helical nature of the edge states, and therefore a finite and polarized heat current is obtained in this configuration. The presence of $e-e$ interchannel interactions strongly affects the current signal, lowering the degree of polarization of the system. Finally, we also show that separate heat and charge flows can be achieved, varying the amplitude of the external gate.
\end{abstract}

DOI: 10.1103/PhysRevB.95.115412

\section{INTRODUCTION}

The fast development in nanotechnologies has spurred the investigation of quantum effects in electronic devices at the submicron scale [1-5]. In past years, this attention has been devoted mostly to the study of charge transport and related phenomena in electronic nanocircuits [6-11]. Here, spectacular effects have been predicted and also experimentally observed, including quantum interference patterns [1], single electron injection and control in quantum conductors $[7,8,12,13]$, and coherent behavior in hybrid devices $[4,5,9,14-16]$.

Conversely, the question how thermodynamic aspects such as heat transport, power conversion, and energy exchange work at the nanoscale has posed interesting challenges [14,17-23]. A precise control and manipulation of heat flows in quantum conductors can lead to interesting applications, i.e., new logic devices based purely on thermal transport. Towards this goal, pioneering experiments have achieved the phase control of coherent thermal transport in hybrid quantum systems [5,14,24-27].

In this respect, the possibility to tune and control some physical parameters by means of external fields and a timedependent potential is of great interest [28-33]. Indeed, a periodically driven system can be immediately associated with thermal machines or heat engines which are based on cyclic operations. Different papers focused on the effects induced by an external driving field both in closed and open quantum systems. For example, the influence of an ac field on the efficiency of thermoelectric setups or heat pumping mechanisms on quantum-dot-based devices have been investigated [28,29,31,34-36].

The dynamical aspects of time-dependent energy and heat flows have been addressed in order to understand fundamental thermodynamical problems such as energy exchange, work distribution, and entropy production (see reviews $[20,31]$ and references therein).

Interestingly, locally applied time-dependent voltages can lead to heat pumping mechanisms in quantum systems
[9,31,37-42]. The peculiar characteristic of heat pumps is that a direct heat current is generated by a purely ac drive which acts against some present thermal gradients. Moreover, it can also generate dc finite current signals even in a purely equilibrium situation, in the absence of any thermal or voltage bias $[31,43,44]$.

Recently, much attention has also been paid to the interplay between heat and spin transport properties, in the emerging research field of spin caloritronics [45-47]. Thermally driven spin sources are very interesting for the improvement of spintronic devices, which are believed to be more efficient with respect to their electronic counterparts [14,20,48]. Striking experimental results in this field have extended the thermoelectric concept to spin transport by the observation of spin-Seebeck [49] and spin-Peltier [50] effects in magnetic systems. Intriguingly, spin-dependent effective temperatures of the electrons were observed in nanopillar spin valves powered by a heat source, thus showing that electron heat conduction can depend on the spin degree of freedom [51,52].

Among all mesoscopic systems for the investigation of spin transport properties, a relevant role is certainly played by two-dimensional topological insulators (2D TIs) [53-56]. These system are constituted by a wide bulk gap and charge and energy transport are mediated by the presence of edge states. These are constituted by two counterpropagating electronic states with opposite spin polarization (spin-momentum locking), and are also called helical edge states [53,57]. Moreover, as long as time-reversal symmetry is preserved and multiparticle inelastic backscattering is absent [58], electrons flowing along these edge states are topologically protected against backscattering and transport occurs in the so-called ballistic regime [53-55]. Experimental evidence of 2D TIs was reported in $\mathrm{CdTe} / \mathrm{HgTe}$ [59] and InAs/GaSb [60,61] quantum wells and has been predicted for a large class of new materials which relies on the presence of strong spin-orbit coupling [62-64]. These systems constitute an interesting playground to study coherent heat transport in which the spin degree of freedom can play a nontrivial role $[31,65]$. Moreover, the unavoidable 
presence of $e-e$ interactions in helical edge states [53,66-69] can dramatically affect the dynamics and have important consequences on thermal transport properties [65].

In this paper we focus exactly on these issues. We consider a 2D TI device in a double quantum point contact (QPC) geometry [70-77]. In this setup, tunneling of electrons is allowed by the presence of constrictions and can lead to quantum interference effects. We also introduce a model to couple electrons of the top edge to a local time-dependent gate potential located above a single QPC. Our aim is to investigate the generation of a direct heat current by the time-dependent drive along the edge of a 2D TI. We demonstrate that the presence of an ac field can generate dc finite current signals in the absence of thermal or voltage bias, relying on a quantum pumping mechanism. We inspect the interplay with the peculiar spin properties of helical edge states and heat transport. We do not take into account a spin-non-conserving mechanism in our model, such as the one due to Rashba scatterers, since here we concentrate on the role played by $e-e$ interactions in the helical edge states. We show that in the proposed setup a polarized heat current can be ensured, carried solely by a single spin species. Moreover, the presence of $e-e$ interactions can strongly modify the behavior of the heat pumped current in this device. Interestingly, it turns out that by looking at the resulting oscillating patterns, one can identify the presence of $e-e$ interactions in the system.

The paper is organized as follows. In Sec. II, we present the setup and describe the various contributions to the Hamiltonian of the system. In Sec. III, we define charge and heat currents and evaluate their average values. Section IV is devoted to a discussion of our main results. Finally, in Sec. V, we draw our conclusions.

\section{MODEL AND SETUP}

We consider a 2D TI connected to two reservoirs at equilibrium kept at the same chemical potential $\mu$ and temperature $T$.

Here, electrons can propagate along the edges and, in the absence of interactions, have well-defined chirality of right$(R)$ and left- $(L)$ moving particles [53,54]. Moreover, the spin-momentum locking property of $2 \mathrm{D}$ TI constrains the direction of propagation and the spin projection of electrons: In the top edge the spin of $R$ electrons is $\uparrow$ and the spin of $L$ electrons is $\downarrow$, and vice versa for the bottom edge. These edge states are usually referred to as helical liquids (HLLs), and in the following we assume that there are no magnetic impurities or Rashba scatterers [58,78-80] close to the edges which can lead to spin-non-conserving processes along the edge itself. Electrons in the top edge are capacitively coupled to an external gate potential $V_{g}(x, t)$, confined in a region $-L_{2}<x<L_{1}$ (see Fig. 1). The combination of the asymmetry and of the time-dependent gate potential, assumed to be periodic in time for simplicity, is crucial in order to generate finite dc current signals, in the absence of a dc voltage bias [31,41]. Top and bottom edges are separated by macroscopic distances and interedge tunneling is only possible in the presence of some constrictions. Here, we consider a double quantum point contact (QPC) geometry [72-74], where the two constrictions are placed at $x= \pm d$, as schematically shown in Fig. 1. The

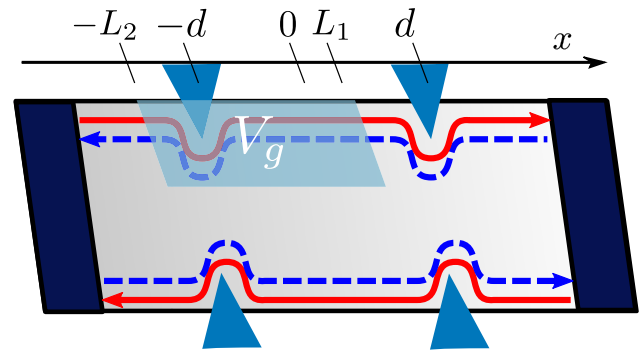

FIG. 1. Scheme of the proposed setup. A 2D TI is connected to two reservoirs with chemical potential $\mu$ and temperature $T$. On each edge, the direction of propagation of spin $\uparrow$ (solid red line) and spin $\downarrow$ (dashed blue line) electrons is opposite. Two quantum point contacts, placed at $x= \pm d$, allow for interedge tunneling. An ac external gate voltage $V_{g}(x, t)$ is capacitively coupled to the top edge in the region around $x \sim-d$ between $-L_{2}$ and $L_{1}$ ( $L_{1}$ can take a value between $\left.-d<L_{1}<d\right)$.

total system can be described by the following Hamiltonian,

$$
\hat{H}=\hat{H}_{\mathrm{HLL}}+\hat{H}_{\text {tun }}+\hat{H}_{g},
$$

where $\hat{H}_{\mathrm{HLL}}=\hat{H}_{\text {free }}+\hat{H}_{\text {int }}$ describes the interacting edge states of the 2D TI. The free contribution associated with fermionic channels can be written as

$$
\hat{H}_{\text {free }}=\sum_{r=R / L} \sum_{\sigma=\uparrow, \downarrow} \hat{H}_{r, \sigma}
$$

with (from now on, $\hbar=1$ )

$$
\hat{H}_{r, \sigma}=-i \xi_{r} v_{\mathrm{F}} \int d x e^{-i \xi_{r} k_{\mathrm{F}} x}: \hat{\psi}_{r, \sigma}^{\dagger}(x) \partial_{x}\left[e^{i \xi_{r} k_{\mathrm{F}} x} \hat{\psi}_{r, \sigma}(x)\right]
$$

where $v_{\mathrm{F}}$ and $k_{\mathrm{F}}=\mu / v_{\mathrm{F}}$ are the Fermi velocity and Fermi momentum, respectively, $\xi_{R / L}= \pm 1$, and $\hat{\psi}_{r, \sigma}^{\dagger}(x)$ the corresponding electron creation operator. In the following, electron interactions on each edge are treated assuming the breaking of Galilean invariance, i.e., in the absence of intrachannel interactions $[3,4,57,81,82]$, and the corresponding term reads

$$
\hat{H}_{\text {int }}=\sum_{\sigma=\uparrow, \downarrow} 2 g_{2} \int d x \hat{\rho}_{R, \sigma}(x) \hat{\rho}_{L,-\sigma}(x),
$$

where $g_{2}$ is the coupling constant describing the interchannel interaction strength (for the sake of convenience, we have adopted the same label as in the Luttinger liquid language [3]). In the above equation the density operator is denoted as $\hat{\rho}_{r, \sigma}(x)=: \hat{\psi}_{r, \sigma}^{\dagger}(x) \hat{\psi}_{r, \sigma}(x)$ :. Following standard bosonization prescriptions [3,4,81] (see Appendix A for more details), $\hat{H}_{\mathrm{HLL}}$ can be written in a diagonal form in terms of chiral bosonic field operators $\hat{\phi}_{ \pm}^{(\ell)}(x)$ as

$$
\hat{H}_{\mathrm{HLL}}=\sum_{\ell=1,2} u \int d x\left\{\left[\partial_{x} \hat{\phi}_{+}^{(\ell)}(x)\right]^{2}+\left[\partial_{x} \hat{\phi}_{-}^{(\ell)}(x)\right]^{2}\right\},
$$

where $u=v_{\mathrm{F}} \frac{2 K}{1+K^{2}}$ represents the renormalized velocity, expressed in terms of the dimensionless interaction parameter $K=\sqrt{\frac{2 \pi v_{\mathrm{F}}-g_{2}}{2 \pi v_{\mathrm{F}}+g_{2}}}$ [82] and $\ell=1,2$ identifies the top/bottom edge, respectively. It is worth noting that in this work we restrict the analysis to the range of interaction strengths given 
by $1 / \sqrt{3} \leqslant K \leqslant 1$, for which it is possible to show that single electron tunneling is the dominant process (in the renormalization group sense), both in single and double QPC geometry $[57,65,73,74]$.

In this work, we assume that the external gate potential $V_{g}(x, t)$ is capacitively coupled to the electron density of the top edge via

$$
\hat{H}_{g}=-e \int d x V_{g}(x, t)\left[\hat{\rho}_{R, \uparrow}(x)+\hat{\rho}_{L, \downarrow}(x)\right],
$$

with the time-dependent potential

$$
V_{g}(x, t)=\left[\theta\left(x+L_{2}\right)-\theta\left(x-L_{1}\right)\right] V(t) .
$$

Here, $L_{1}, L_{2}$ are linked to the dimension of the gate (see Fig. 1), and $V(t)=V_{0} \cos (\omega t)$ is a periodic time-dependent drive, with period $\mathcal{T}=\frac{2 \pi}{\omega}$. In particular, we will consider the gate located around $x \sim-d$, i.e., $-L_{2}<-d\left(L_{2}>0\right)$ and $-d<L_{1}<d$, as shown in Fig. 1. We point out that a generic time-dependent potential $V_{g}(x, t)$ will break time-reversal invariance, depending on the precise shape of the potential $V_{g}(x, t)$. We underline that the chosen shape of $V(t)$ preserves time-reversal symmetry.

Tunneling events between the two edges are allowed in the double QPC geometry under consideration. For the sake of simplicity, here we focus on spin-preserving tunneling events described by [69-72]

$$
\hat{H}_{\text {tun }}=\sum_{\sigma=\uparrow, \downarrow} \int d x h(x) e^{i 2 k_{\mathrm{F}} x} \hat{\psi}_{L, \sigma}^{\dagger}(x) \hat{\psi}_{R, \sigma}(x)+\text { H.c., }
$$

with $h(x)=\Lambda \sum_{p= \pm 1} \delta(x-p d)$ the space-dependent amplitude describing the double constrictions depicted in Fig. 1. Here, $\Lambda$ represents the tunneling amplitude which is assumed to be energy and time independent, as usually discussed in closely related experimental geometry [8]. In the following analysis we do not include spin-flipping tunneling at the QPCs: since the bare amplitudes of the tunneling processes are usually assumed to be unmodified by the presence of a time-dependent gate voltage, we expect the amplitude of spin-flipping tunneling to be smaller with respect to the spinpreserving one $[70,83]$. Moreover, we note that, since in our model the spin quantization axes are a good quantum number, spin-non-conserving mechanisms, such as magnetic impurities or Rashba scatterers [58,79,80,84], are not supposed to affect the tunneling dynamics. Finally, we note that the chosen configuration, with the external gate located around a single QPC at $x \sim-d$, naturally guarantees the required asymmetry needed for a quantum pumping mechanism $[9,40,41]$.

\section{AVERAGE PUMPED CURRENTS}

We are interested in the study of transport properties in the setup introduced above. We recall that the 2D TI is connected to two reservoirs, which we assume to be at equilibrium at the same chemical potential $\mu$ and same temperature $T$. Nevertheless, finite dc current signals can be generated due to the presence of the time-dependent gate potential. Indeed, as already mentioned, the ac field supplied by the external gate above one single QPC can lead to a quantum pumping mechanism, which, in turn, generates dc finite currents. Here, we investigate this aspect by studying the pumped currents which flow through the 2D TI. In particular, we will consider the generation of heat current $I_{q}$ and charge current $I_{c}$, focusing on their dc components, extracted from the finite temperature current contributions by further averaging the signals over one period of the gate potential $V(t)$.

It is possible to show (see Appendix A) that in the dc limit the only possible contributions to pumped currents are due to backscattering. The latter are present whenever the edges are coupled and tunneling events occur, i.e., in proximity to the two QPCs. Therefore, in this section we focus on the evaluation of the backscattering contribution to the average currents (we thus introduce a related index BS). Backscattering charge current $\hat{I}_{c}^{\mathrm{BS}}$ can be defined as

$$
\hat{I}_{c}^{\mathrm{BS}} \equiv-e\left(\hat{I}_{N \uparrow}^{\mathrm{BS}}+\hat{I}_{N \downarrow}^{\mathrm{BS}}\right),
$$

where $(-e)$ is the electron charge $(e>0)$ and we have identified the particle current per spin component, which is given by

$$
\hat{I}_{N \sigma}^{\mathrm{BS}}=\frac{i}{2}\left[\hat{H}_{\mathrm{tun}}, \hat{N}_{R, \sigma}-\hat{N}_{L, \sigma}\right],
$$

with the particle number operator $\hat{N}_{r, \sigma}=\int d x \hat{\rho}_{r, \sigma}(x)$. Using Eq. (8) we can thus write

$$
\hat{I}_{N \sigma}^{\mathrm{BS}}=i \int d x h(x) e^{i 2 k_{\mathrm{F}} x} \hat{\psi}_{L, \sigma}^{\dagger}(x) \hat{\psi}_{R, \sigma}(x)+\text { H.c. }
$$

Analogously, the backscattering heat current flowing between the two reservoirs can be written as the sum of the heat current per spin direction,

$$
\hat{I}_{q}^{\mathrm{BS}} \equiv \hat{I}_{q \uparrow}^{\mathrm{BS}}+\hat{I}_{q \downarrow}^{\mathrm{BS}}
$$

with

$$
\hat{I}_{q \sigma}^{\mathrm{BS}} \equiv \frac{i}{2}\left[\hat{H}_{\mathrm{tun}}, \hat{Q}_{R, \sigma}-\hat{Q}_{L, \sigma}\right],
$$

where $\hat{Q}_{r, \sigma}=\hat{H}_{r, \sigma}-\mu \hat{N}_{r, \sigma}$. The expression in Eq. (13), related to the heat current contribution per spin component, in terms of fermionic fields is

$$
\hat{I}_{q \sigma}^{\mathrm{BS}}=\frac{v_{\mathrm{F}}}{2} \int d x h(x) e^{i 2 k_{\mathrm{F}} x} \partial_{x}\left[\hat{\psi}_{L, \sigma}^{\dagger}(x) \hat{\psi}_{R, \sigma}(x)\right] .
$$

It is worth underlining that Eq. (12) corresponds to the net amount of heat flow exchanged between the $R$ channel and $L$ channel due to backscattering processes and it does not give any information on the heat which locally enters into each reservoir separately.

Using the standard perturbative approach $[4,65,72,85]$ in the tunneling Hamiltonian, the average pumped currents can be calculated. At the lowest order in the tunneling one has the finite temperature currents (here, $v=n, q$ for particle-number and heat contributions)

$$
I_{v \sigma}^{\mathrm{BS}}=i \int d t^{\prime} \theta\left(t-t^{\prime}\right)\left\langle\left[\hat{H}_{\mathrm{tun}}^{(0)}\left(t^{\prime}\right), \hat{I}_{v \sigma}^{\mathrm{BS},(0)}(t)\right]\right\rangle_{0},
$$

where the average $\langle\cdots\rangle_{0}$ is taken with respect to $\hat{H}^{\mathrm{HLL}}+\hat{H}_{g}$

In the above equation we have introduced the index (0) to indicate the time evolution of operators evaluated in the absence of tunneling, i.e., with respect to $\hat{H}_{\mathrm{HLL}}+\hat{H}_{g}$ for the top edge and to $\hat{H}_{\mathrm{HLL}}$ for the bottom one. We remind the reader 
that in our model the external gate potential couples only to the top edge electrons (see Fig. 1). In order to evaluate the expression in Eq. (15), it is sufficient to know the time evolution of fermionic operators, obtained from $\hat{H}_{\mathrm{HLL}}+\hat{H}_{g}$. In particular, we are interested only in their time evolution close to the QPC positions at $x= \pm d$, since we are assuming local tunneling with a $\delta$-like spatial shape for the QPCs centered around $x= \pm d$. The explicit derivation of the time evolution of these operators is reported in Appendix B for the case of a sinusoidal voltage $V(t)=V_{0} \cos (\omega t)$, and here we quote only the relevant results:

$$
\begin{aligned}
& \hat{\psi}_{R, \uparrow}(x, t)=e^{i A_{1 \uparrow} \cos \left[\omega t-\theta_{1 \uparrow}(x)\right]} \hat{\psi}_{R, \uparrow}^{(\mathrm{HLL})}(x, t), \\
& \hat{\psi}_{L, \downarrow}(x, t)=e^{i A_{1 \downarrow} \cos \left[\omega t-\theta_{1 \downarrow}(x)\right]} \hat{\psi}_{L, \downarrow}^{(\mathrm{HLL})}(x, t),
\end{aligned}
$$

in the region close to the QPC1 (right) at $x=d$, and

$$
\begin{aligned}
& \hat{\psi}_{R, \uparrow}(x, t)=e^{i A_{2 \uparrow}(x) \cos \left[\omega t-\theta_{2 \uparrow}(x)\right]} \hat{\psi}_{R, \uparrow}^{(\mathrm{HLL})}(x, t), \\
& \hat{\psi}_{L, \downarrow}(x, t)=e^{i A_{2 \downarrow}(x) \cos \left[\omega t-\theta_{2 \downarrow}(x)\right]} \hat{\psi}_{L, \downarrow}^{(\mathrm{HLL})}(x, t),
\end{aligned}
$$

in the region around the QPC2 (left) located at $x=-d$. In the above equations we have denoted with $\hat{\psi}_{r, \sigma}^{(\mathrm{HLL})}(x, t)$ the time evolution of operators with respect to the bare $\hat{H}_{\mathrm{HLL}}$, without the external gate. The amplitudes and phases introduced just above are given by

$$
\begin{gathered}
A_{2 \sigma}(x)=\frac{e V_{0}}{2 \omega} \sqrt{\alpha_{\sigma}^{2}(x)+\beta_{\sigma}^{2}(x)}, \\
\theta_{2 \sigma}(x)=\arctan \frac{\alpha_{\sigma}(x)}{\beta_{\sigma}(x)}+2 m \pi \quad(\text { with } m \in \mathbb{N}), \\
A_{1 \uparrow / 1 \downarrow}=\frac{e V_{0}}{\omega}(1 \pm K) \sin \left[\frac{\omega}{2 u}\left(L_{2}+L_{1}\right)\right], \\
\theta_{1 \sigma}(x)=\frac{\omega}{2 u}\left(L_{2}-L_{1}+2 x\right),
\end{gathered}
$$

where the amplitudes $A_{1 \sigma}$ have no spatial dependence, contrary to what happens for $A_{2 \sigma}(x)$ which is affected by the gate voltage $V_{g}$. The coefficients $\alpha_{\sigma}(x)$ and $\beta_{\sigma}(x)$ are connected to the geometry of the setup and are given by

$$
\begin{aligned}
\alpha_{\uparrow / \downarrow}(x)= & 1-\cos \left[\omega\left(\frac{L_{2}+L_{1}}{2 u}\right)\right] \cos \left[\omega\left(\frac{x}{u}+\frac{L_{2}-L_{1}}{2 u}\right)\right] \\
& \pm K \sin \left[\omega\left(\frac{L_{2}+L_{1}}{2 u}\right)\right] \sin \left[\omega\left(\frac{x}{u}+\frac{L_{2}-L_{1}}{2 u}\right)\right], \\
\beta_{\uparrow / \downarrow}(x)= & \sin \left[\omega\left(\frac{L_{2}+L_{1}}{2 u}\right)\right] \cos \left[\omega\left(\frac{x}{u}+\frac{L_{2}-L_{1}}{2 u}\right)\right] \\
& \pm K \cos \left[\omega\left(\frac{L_{2}+L_{1}}{2 u}\right)\right] \sin \left[\omega\left(\frac{x}{u}+\frac{L_{2}-L_{1}}{2 u}\right)\right] .
\end{aligned}
$$

One can immediately note that in the noninteracting case, i.e., $K=1$, the amplitude $A_{1 \downarrow}=0$ vanishes. This will have significant implications on the pumped charge and heat currents, as we will discuss below.

We recall that the tunneling is local at the QPC positions, with amplitude $h(x)=\Lambda[\delta(x-d)+\delta(x+d)]$, and we introduce the following series representation [86,87],

$$
\begin{aligned}
h(x) e^{i A_{1 \sigma / 2 \sigma}(x) \cos \left[\omega t-\theta_{1 \sigma / 2 \sigma}(x)\right]} & \\
= & \Lambda \sum_{n=0}^{\infty}(i)^{n}\left(2-\delta_{n, 0}\right) \delta(x \mp d) J_{n}\left(A_{1 \sigma / 2 \sigma}(x)\right) \\
& \times \cos \left[n\left(\omega t-\theta_{1 \sigma / 2 \sigma}(x)\right)\right],
\end{aligned}
$$

where $J_{n}(x)$ is the Bessel function of $n$th order. Notice that the first order of the above Fourier expansion is consistent with the periodic modulation of the tunneling amplitudes introduced by hand in Refs. [72,86]. By inserting this relation into Eq. (15) one can obtain the expressions for the average pumped currents. We stress that the only contributions come from positions located at the QPCs $x= \pm d$. We thus introduce the simplified notations

$$
\begin{aligned}
& A_{2 \sigma}(x=-d) \equiv A_{2 \sigma} \quad \theta_{1 \sigma}(x=d) \equiv \theta_{1 \sigma}, \\
& \theta_{2 \sigma}(x=-d) \equiv \theta_{2 \sigma},
\end{aligned}
$$

and we obtain the dc components,

$$
\begin{aligned}
I_{N \sigma}^{\mathrm{BS}}= & 2 i \frac{|\Lambda|^{2}}{(\pi a)^{2}} \sum_{n=1}^{\infty} J_{n}\left(A_{1 \sigma}\right) J_{n}\left(A_{2 \sigma}\right) \sin \left[n\left(\theta_{2 \sigma}-\theta_{1 \sigma}\right)\right] \\
& \times \sin \left(4 k_{\mathrm{F}} d\right) \int d \tau \sin (n \omega \tau) P_{\gamma}\left(\tau-\frac{2 d}{u}\right) P_{\gamma}\left(\tau+\frac{2 d}{u}\right),
\end{aligned}
$$

for the average particle current per spin component, and

$$
\begin{aligned}
I_{q \sigma}^{\mathrm{BS}}= & i \frac{|\Lambda|^{2}}{(\pi a)^{2}} \frac{v_{\mathrm{F}}}{u} \sum_{n=1}^{\infty} J_{n}\left(A_{1 \sigma}\right) J_{n}\left(A_{2 \sigma}\right) \sin \left[n\left(\theta_{2 \sigma}-\theta_{1 \sigma}\right)\right] \\
& \times \cos \left(4 k_{\mathrm{F}} d\right) \int d \tau \sin (n \omega \tau)\left[\partial_{\tau} P_{\gamma}\left(\tau-\frac{2 d}{u}\right)\right. \\
& \left.\times P_{\gamma}\left(\tau+\frac{2 d}{u}\right)-P_{\gamma}\left(\tau-\frac{2 d}{u}\right) \partial_{\tau} P_{\gamma}\left(\tau+\frac{2 d}{u}\right)\right],
\end{aligned}
$$

for the contribution associated with the heat current per spin component. Notice that the $n=0$ contributions in the infinite sums cancel out and the series expressions presented in Eqs. (27) and (28) start from $n=1$. In the above equations we have introduced $a$, the usual short-distance cutoff of HLL [4], and $P_{g}(t)=e^{g \mathcal{W}(t)}$, where $\mathcal{W}(t)$ is the bosonic correlation function $[4,10,88-90]$

$$
\mathcal{W}(t)=\ln \frac{\left|\Gamma\left(1+\frac{k_{\mathrm{B}} T}{\omega_{\mathrm{c}}}+i k_{\mathrm{B}} T t\right)\right|^{2}}{\Gamma^{2}\left(1+\frac{k_{\mathrm{B}} T}{\omega_{\mathrm{c}}}\right)\left(1+i \omega_{\mathrm{c}} t\right)} .
$$

Here, $\omega_{\mathrm{c}}=v_{\mathrm{F}} / a$ is the energy cutoff, which represents the highest energy scale of the problem. Note that this quantity can be related to the energy gap between the bulk conduction and valence bands of the 2D TI. The dimensionless parameter $\gamma$ depends on the interaction strength and reads

$$
\gamma \equiv \frac{1}{2}\left(\frac{1}{K}+K\right) \text {. }
$$


Performing the integrals present in Eqs. (27) and (28) (see Ref. [73] and Appendix C for details) the average pumped currents can be written as

$$
\begin{aligned}
I_{N \sigma}^{\mathrm{BS}}= & 4|\lambda|^{2} \sum_{n=1}^{\infty} J_{n}\left(A_{1 \sigma}\right) J_{n}\left(A_{2 \sigma}\right) \sin \left[n\left(\theta_{2 \sigma}-\theta_{1 \sigma}\right)\right] \\
& \times \sin \left(4 k_{F} d\right) \mathcal{H}\left(\gamma, \frac{2 d n \omega}{u}, \frac{2 d k_{B} T}{u}\right) \\
& \times\left[\tilde{\mathcal{P}}_{2 \gamma}(n \omega)-\tilde{\mathcal{P}}_{2 \gamma}(-n \omega)\right] \\
I_{q \sigma}^{\mathrm{BS}}= & -v_{\mathrm{F}}|\lambda|^{2} \sum_{n=1}^{\infty} J_{n}\left(A_{1 \sigma}\right) J_{n}\left(A_{2 \sigma}\right) \sin \left[n\left(\theta_{2 \sigma}-\theta_{1 \sigma}\right)\right] \\
& \times \cos \left(4 k_{F} d\right) \Xi\left(\gamma, 2 d, \frac{n \omega}{u}, \frac{k_{B} T}{u}\right) \\
& \times\left[\tilde{\mathcal{P}}_{2 \gamma}(n \omega)-\tilde{\mathcal{P}}_{2 \gamma}(-n \omega)\right],
\end{aligned}
$$

where $\lambda=\Lambda /(2 \pi a)$ and $\mathcal{H}$ and $\Xi$ are modulating functions (see Appendix $\mathrm{C}$ for the explicit expressions) and $\tilde{\mathcal{P}}_{g}(E)$ is the Fourier representation in the energy domain of $\mathcal{P}_{g}(t)$. In passing, we note that $\mathcal{H}$ and $\Xi$ do not depend on the voltage amplitude $V_{0}$, but this dependence is crucially present in the argument of the Bessel functions. Before discussing the behavior of the average pumped currents, some comments are in order. First of all, contrary to previous works [72,73], here the time-dependent tunneling amplitudes and phase shifts of the two QPCs are not introduced a priori in the system. Indeed, the solution of the microscopic model of the coupling to the external gate have lead to the time dependence of the tunneling amplitude and to the microscopic derivation of the phase shifts $\theta_{1 \sigma}$ and $\theta_{2 \sigma}$. One can note that dc finite current signals strongly rely on quantum interference effects $[14,65,75]$, induced by the presence of the two constrictions in this geometry. Indeed, both currents in Eqs. (31) and (32) vanish in the limit $d \rightarrow 0$. From a physical point of view, each contribution in the sum over $n$ accounts for tunneling processes in which electrons absorb or emit $n$ photons. Therefore, the tunneling amplitudes, for each QPC, associated with these processes are weighted by the corresponding Bessel function $J_{n}(x)$ present in Eq. (25).

We also note that quantum interference effects are suppressed if the tunneling amplitude through the left $(x=-d)$ QPC $A_{2 \sigma}$ or through the right $(x=d)$ QPC $A_{1 \sigma}$ vanish $A_{1 \sigma / 2 \sigma} \rightarrow 0$, resulting in a vanishing pumped current signal. The fact that both currents strongly rely on quantum interference implies that the probability of propagation across the junctions for electrons is related to the phase acquired in the tunneling process. These phases depend on the energy at which electrons impinge upon the barrier and on the phase shift $\Delta \theta_{\sigma}=\theta_{2 \sigma}-\theta_{1 \sigma}$ between the two QPCs. Since the phase shift $\Delta \theta_{\sigma}$ has opposite signs for $R, \sigma$ and $L, \sigma$ electrons, it generates a left-right asymmetry which is crucial for the generation of currents (31) and (32) [91]. This asymmetry, together with the energy dependence of the tunneling junctions, entails that, at each energy, electrons moving in a specific direction are more likely to be transmitted across the junctions than electrons moving in the opposite one. This fact is consistent with the general prescription of the parametric pumping mechanism [9,40,41,86,87]. Once Eqs. (31) and (32) have been obtained, all stationary currents can be computed by using the relations in Eqs. (9) and (12).

\section{RESULTS AND DISCUSSION}

In this section we will discuss our main results. We will mainly focus on the generation of finite heat current induced by the pumping mechanism in the absence of external bias. We investigate the net amount of heat current flowing through the 2D TI, studying the behavior of $I_{q}^{\mathrm{BS}}$ as a function of various external parameters, inspecting also the role played by $e-e$ interactions.

In the following, all energies are rescaled with respect to the chemical potential $\mu$ (dimensionless quantities are thus indicated with an overbar, i.e., $\bar{\omega}=\omega / \mu, \bar{V}_{0}=e V_{0} / \mu, \bar{T}=$ $\left.k_{\mathrm{B}} T / \mu, \bar{\omega}_{\mathrm{c}}=\omega_{\mathrm{c}} / \mu\right)$. For the sake of convenience, we also introduce

$$
\eta=d k_{\mathrm{F}}=d \frac{\mu}{v_{\mathrm{F}}}
$$

a dimensionless parameter directly connected to the presence of quantum interference effects, as it appears in the expressions for the pumped current, Eqs. (31) and (32), and is linearly proportional to the distance between the two QPCs.

We restrict the analysis to a physically reasonable range of parameters, remaining as close as possible to experimentally accessible values. We fix the chemical potential to $\mu=3 \mathrm{meV}$ and the temperature to $T=300 \mathrm{mK}(\bar{T}=0.01)$. The energy cutoff, which represents the largest energy scale involved, is related to the typical energy bulk gap in $2 \mathrm{D}$ TI. Recent proposals have predicted a very wide bulk gap for 2D TIs in topological materials with strong spin-orbit coupling [62-64]. Recent measurements on novel devices have reported bulk gap values up to $\sim 800 \mathrm{meV}$ [62]. Here we fix the energy cutoff to $\bar{\omega}_{\mathrm{c}}=200$.

Concerning the characteristic parameters of the external gate, here we fix $\bar{\omega}=0.1$, which corresponds to a drive frequency of $v=\omega /(2 \pi) \sim 75 \mathrm{GHz}$. We vary the gate amplitude in the range $0 \leqslant \bar{V}_{0} \leqslant 20$. In this case, we can investigate a large range of parameters, including the nonlinear regime $e V_{0} / \omega \gg 1$ where interesting features appear.

Let us start considering the noninteracting case $(K=1)$. Interestingly enough, we get $A_{1 \downarrow}=0$, which in turn leads to the vanishing of the heat current component associated with $\downarrow$ electrons $I_{q \downarrow}^{\mathrm{BS}}=0$. This fact leads to the conclusion that, in the noninteracting case, a finite heat current is produced and is associated only with a single spin species $\sigma=\uparrow$,

$$
\lim _{K=1} I_{q}^{\mathrm{BS}}=I_{q \uparrow}^{\mathrm{BS}} \text {. }
$$

Therefore, this setup can allow for the generation of fully polarized heat current. Note that the vanishing of the component associated with $\downarrow$ electrons with $A_{1 \downarrow} \rightarrow 0$ is intimately connected to the spin-momentum locking property of $2 \mathrm{D}$ TIs. In the end this is also connected to the chosen geometry. Indeed, if the external gate is placed on top of the other QPC, this would imply $A_{2 \uparrow} \rightarrow 0$ with an opposite polarization of the pumped heat current. Indeed, physically, the vanishing of spin $\downarrow$ components can be understood in terms of the tunneling paths of electrons. When $L, \downarrow$ electrons reach the right QPC, they have not increased or reduced their energy 


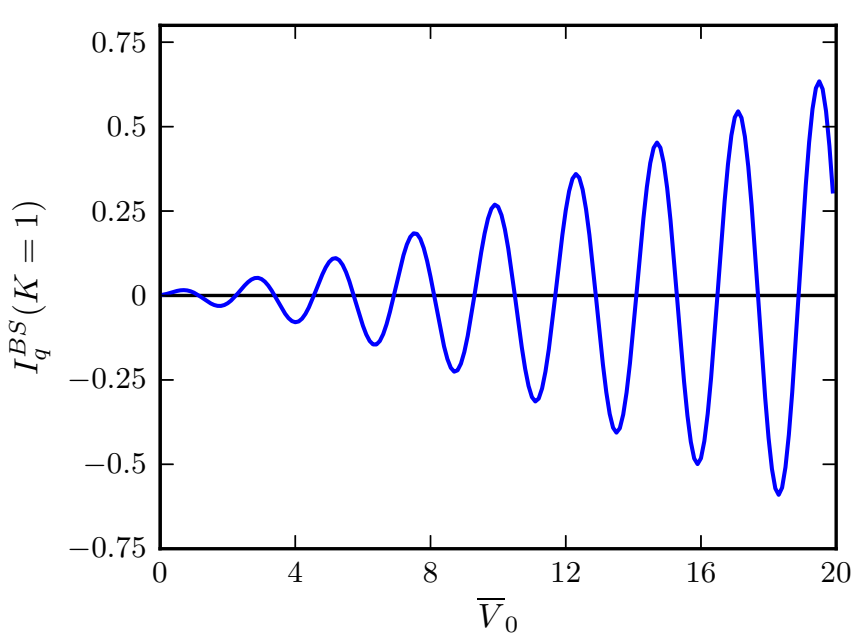

FIG. 2. Average pumped heat current $I_{q}^{\mathrm{BS}}$ (in units of $10^{-3}\left|\lambda^{2}\right| / \bar{\omega}_{\mathrm{c}}$ ) as a function of the external gate voltage $\bar{V}_{0}=e V_{0} / \mu$ in the noninteracting case $(K=1)$. Drive frequency and temperature are fixed to $\bar{\omega}=0.1$ and $\bar{T}=0.01$. Other parameters are $\eta=1.5$, $L_{1}=0.2 d, L_{2}=2 d$, and the energy cutoff is set to $\bar{\omega}_{\mathrm{c}}=200$. As discussed in the text, finite heat current is generated by pumping and for $K=1$ it is only carried by $\uparrow$ electrons, resulting in a flow of polarized heat current.

yet by passing through the gate. Therefore, each contribution to transport that could arise from $L, \downarrow$ tunneling at $x=d$ is exactly compensated by the contribution $R, \downarrow$ tunneling at the right QPC. Only the tunneling path through the other QPC is due to $\downarrow$ electrons and interference effects would completely vanish.

In Fig. 2 we show the average pumped heat current $I_{q}^{\mathrm{BS}}=$ $I_{q \uparrow}^{\mathrm{BS}}$ in the noninteracting case, where an oscillating behavior is clearly visible as a function of the gate voltage $\bar{V}_{0}$. The latter is due to the Bessel functions $J_{n}\left(A_{1 \uparrow}\right)$ and $J_{n}\left(A_{2 \uparrow}\right)$ present in Eq. (32). In order to understand this oscillating behavior, it is instructive to look at the limit of small $\eta \bar{\omega}$ of $A_{1 \uparrow}$ (as it is in our case with the chosen parameters),

$$
\lim _{\eta \bar{\omega}<1} A_{1 \uparrow} \rightarrow \bar{V}_{0} \eta \frac{L_{1}+L_{2}}{d},
$$

proportional to $\bar{V}_{0}$. One can see that for $\eta\left(L_{1}+L_{2}\right) / d>1$, as it is in our case, the maxima and minima of $J_{n}\left(A_{1 \uparrow}\right)$, as a functions of the gate amplitude, are several in the considered range of $\bar{V}_{0}$. This basically would explain the oscillating pattern present in Fig. 2 for the polarized heat current in the noninteracting case. Indeed, the other $J_{n}\left(A_{2 \uparrow}\right)$ present a slower oscillating behavior, since $A_{2 \uparrow}$ is linked to $L_{2}-d$ always smaller than $L_{1}+L_{2}$. We underline that the same qualitative features are present also for higher values of $\bar{\omega} \eta$, where in general the full expression of $A_{1 \uparrow}$ governs the observed pattern. Finally, the magnitude of $I_{q}^{\mathrm{BS}}$ is related to the weight function present in Eq. (32) and to the product of Bessel functions itself. The latter are responsible of the increasing magnitude for increasing gate voltage $\bar{V}_{0}$ in the chosen range of parameters, where Bessel functions of higher order $n$ become relevant.

The presence of $e$ - $e$ interactions strongly modifies the above picture, as we now discuss.
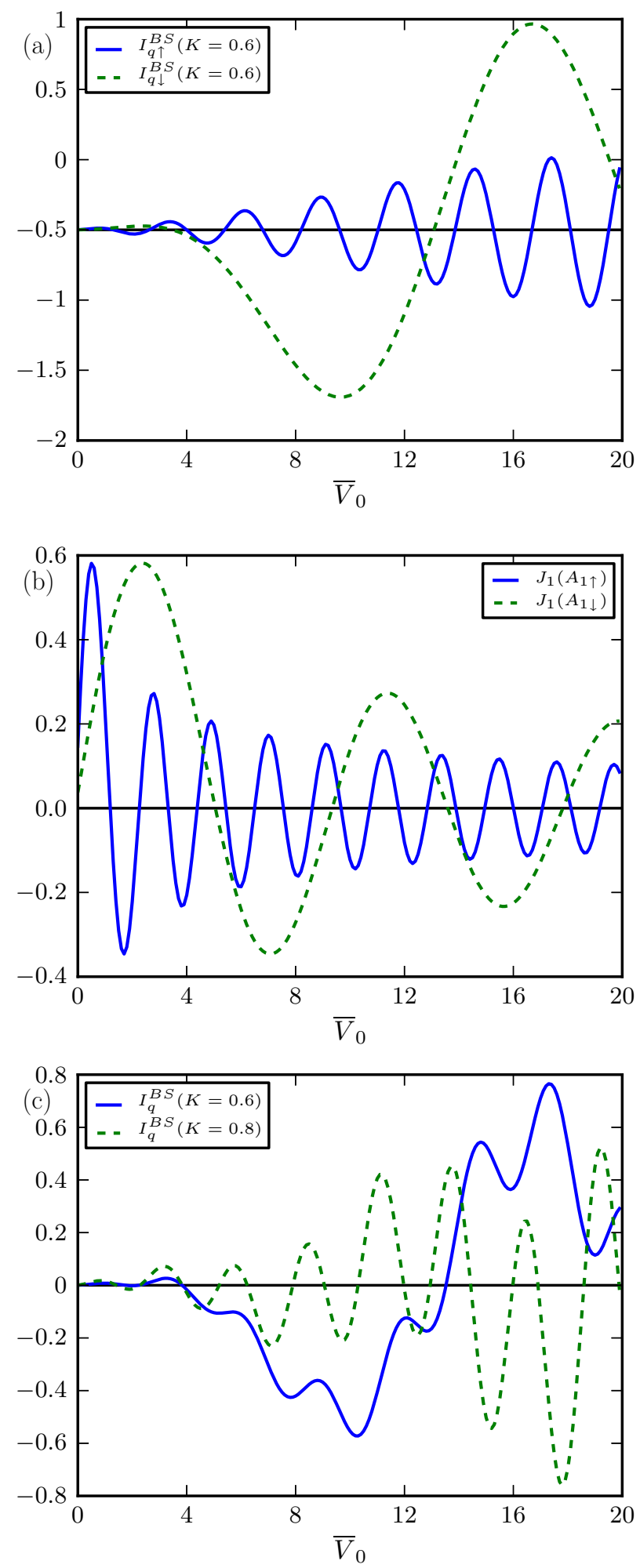

FIG. 3. Average pumped heat current in the presence of $e$-e interactions $(K<1)$. (a) shows the spin-up $I_{q \uparrow}^{\mathrm{BS}}$ (blue solid line) and spin-down $I_{q \downarrow}^{\mathrm{BS}}$ (green dashed line) components of the average heat current for $K=0.6$. (b) represents the behavior of the more relevant Bessel functions with $n=1$ with an interaction strength $K=0.6$. (c) shows the heat current $I_{q}^{\mathrm{BS}}$ (in units of $10^{-3}\left|\lambda^{2}\right| / \bar{\omega}_{\mathrm{c}}$ ) as a function of the gate voltage $\bar{V}_{0}=e V_{0} / \mu$. The blue solid line and green dashed curve correspond to $K=0.6$ and $K=0.8$, respectively. Other parameters are the same as in Fig. 2. 
The interacting case is reported in Fig. 3. The first important difference with respect to the noninteracting case is that now both spin components contribute to the average pumped heat current $I_{q}^{\mathrm{BS}}=I_{q \uparrow}^{\mathrm{BS}}+I_{q \downarrow}^{\mathrm{BS}}$, and thus the heat current is not polarized anymore [see, in particular, Fig. 3(b)].

The behaviors of $I_{q \uparrow}^{\mathrm{BS}}$ and $I_{q \downarrow}^{\mathrm{BS}}$ can be ascribed qualitatively to the difference between the Bessel functions $J_{n}\left(A_{1 \uparrow}\right)$ and $J_{n}\left(A_{1 \downarrow}\right)$. These functions are plotted in Fig. 3(b) versus the gate voltage $\bar{V}_{0}$. As one can see, $J_{n}\left(A_{1 \uparrow}\right)$ displays more oscillations than $J_{n}\left(A_{1 \downarrow}\right)$. Indeed, looking at Eq. (22), one can note that $A_{1 \downarrow}=\epsilon A_{1 \uparrow}$, with $\epsilon=\frac{1-K}{1+K}$, which is $\epsilon<1$ for all interaction strengths in the interval $0<K \leqslant 1$. Therefore, the number of zeros of $J_{n}\left(A_{1 \downarrow}\right)$ are reduced of a factor $\epsilon$ with respect to $J_{n}\left(A_{1 \uparrow}\right)$ as a function of $\bar{V}_{0}$. We underline that this factor $\epsilon$, related to the interaction parameter $K$, arises as a consequence of the particular form for the phases of the bosonized expression for $\hat{\psi}_{R, \uparrow}$ and $\hat{\psi}_{L, \downarrow}$ in Eq. (B9) and is a peculiar property of HLL.

The differences between $A_{1 \uparrow}$ and $A_{1 \downarrow}$ are also reflected in the different amplitudes of the spin components of the heat current. Summing over the higher $n$th order of the Bessel functions and considering the faster oscillation related to $A_{1 \uparrow}$, the amplitude of $I_{q \uparrow}^{\mathrm{BS}}$ decreases with respect to $I_{q \downarrow}^{\mathrm{BS}}$ [see Fig. 3(a)]. This trend is reflected in the total heat current, i.e., the sum of the two spin contributions, which is thus dominated by the slower oscillation associated to $I_{q \downarrow}^{\mathrm{BS}}$, as one can see in Fig. 3(c). This fact is clearer if one considers stronger interactions (see the green dashed line for $K=0.6$ ), where the period of oscillations is dominated by the slow spin $\downarrow$ component, presenting some beats due to the modulation with the fast oscillation associated to $I_{q \uparrow}^{\mathrm{BS}}$. Due to all these different features, the pumped heat current can be also used as a sensitive probe of the presence of $e-e$ interactions. Indeed, as we have shown, the heat current is no longer fully polarized and presents a different pattern of oscillations, with characteristic modulations related to the interaction strength.

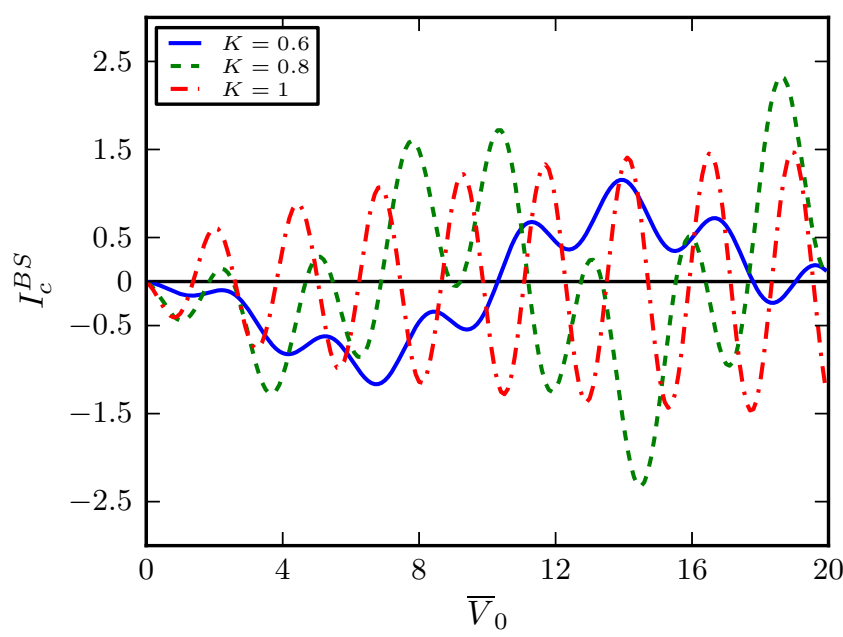

FIG. 4. Pumped charge current $I_{c}^{\mathrm{BS}}$, in units of $4 \times 10^{-4} e \frac{|\lambda|^{2}}{\omega}$, as a function of the gate amplitude $\bar{V}_{0}$. Different curves correspond to different interaction strengths, $K=0.6$ (blue solid line), $K=0.8$ (green dashed line), $K=1$ (red line). Other parameters are the same as in Fig. 2.
A similar behavior is expected also for the average charge current. Indeed, the same pumping mechanism would lead to a finite charge current signal. This is reported in Fig. 4, where $I_{c}^{\mathrm{BS}}$ is plotted as a function of the gate voltage $\bar{V}_{0}$ for different interaction strengths. It turns out that in the noninteracting case the pumped charge current is also fully polarized and carried solely by spin $\uparrow$ electrons. The presence of interactions induces the same qualitative changes discussed for the heat current and the associated oscillations can be explained in full analogy to what happens for $I_{q}^{\mathrm{BS}}$.

It is interesting, however, to look at the sign of the product of the two pumped currents, i.e.,

$$
\mathcal{S}\left(\bar{V}_{0}, K\right) \equiv \frac{I_{c}^{\mathrm{BS}} I_{q}^{\mathrm{BS}}}{\left|I_{c}^{\mathrm{BS}}\right|\left|I_{q}^{\mathrm{BS}}\right|},
$$

as a function of the gate amplitude $\bar{V}_{0}$, as shown in Fig. 5. Here, we show the noninteracting case $K=1$ in the upper panel and $K=0.6$ in the lower panel. In general, the fact that this quantity does not have a definite sign indicates that by
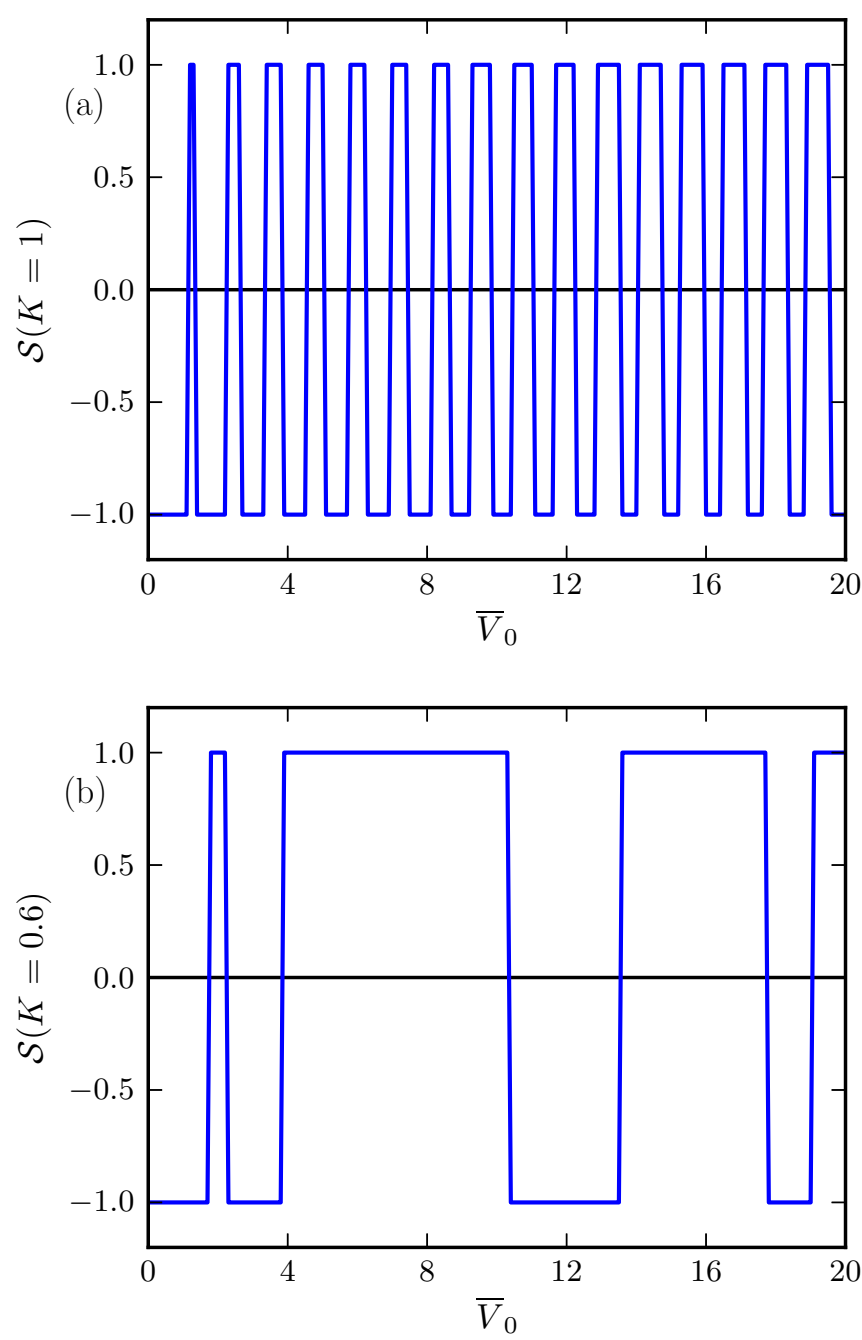

FIG. 5. Sign of the product of charge current $I_{c}^{\mathrm{BS}}$ and heat current $I_{q}^{\mathrm{BS}}$, as a function of the gate amplitude $\bar{V}_{0}$. In the upper panel, $K=1$ (noninteracting case), and in the lower panel, $K=0.6$. Other parameters are the same as in Fig. 2. 
varying the gate voltage one can achieve regimes in which charge and heat currents flow in opposite directions. The difference in the sign of charge and heat currents can be understood in terms of the currents generated by processes with a specific number of absorbed photons $n$. Electrons contributing to these currents belong to an energy window defined by the energy quantum $n \omega$. At each energy within this window, right-moving or left-moving electrons are more likely transmitted, as discussed in Sec. III, resulting, respectively, in positive or negative contributions to the currents. The sum of all these contributions determines the charge and heat currents. However, electrons carry different values of energy across the system and the energy dependence of the tunneling junctions suppresses or increases the transfer of some of them. On the other hand, electrons carry all the same charge. As a consequence, each positive and negative contribution of electrons within the energy window fixed by $n$ is differently weighted for charge and heat. Therefore, this fact leads to the possibility of charge and heat currents generated by a single photoassisted process with opposite sign. Moreover, this holds true also for the total currents $I_{c}$ and $I_{q}$.

In the presence of interactions, when $A_{1 \downarrow} \neq 0$, the lower number of oscillations of $J_{n}\left(A_{1 \downarrow}\right)$ with respect to $J_{n}\left(A_{1 \uparrow}\right)$ allows one to better distinguish the effect of processes of each order $n$ on the sign of charge and heat currents. On the other hand, for $K=1$, only $J_{n}\left(A_{1 \uparrow}\right)$ remains and brings many oscillations into each $n$th process, thus resulting in a strong reduction of the modulating function. In the interacting case (see the lower panel in Fig. 5) the regions with a definite sign of $\mathcal{S}$ as a function of $\bar{V}_{0}$ become wider with respect to the noninteracting one. Here, charge and heat flow in the same-or opposite_-directions for a larger range of the external gate voltage $\bar{V}_{0}$.

\section{CONCLUSIONS}

In this paper, we have investigated a double quantum point contact geometry in a 2D TI. Based on this, we have proposed a setup in which a pumping mechanism can generate finite heat and charge currents without an external dc bias or thermal gradient. The pumping mechanism relies on the presence of an external gate potential placed on top of one of the two constrictions which acts on the system with an external ac field. We have developed a microscopic model of the coupling between the external gate and the electrons of the edge states of the 2D TI. We have therefore evaluated the average heat and charge currents pumped into the system as a function of various parameters, also taking into account the presence of $e-e$ interactions. In the noninteracting case, this setup generates a finite heat current which is carried solely by a single spin species, resulting in a fully polarized heat flow. The presence of $e-e$ interactions strongly modifies the behavior of pumped currents, which are no longer fully polarized since both spin components now contribute to the net heat flow. Moreover, looking at the behavior of the heat current as a function of the gate voltage, one can distinguish between different patterns of oscillations. The latter are connected to the interaction strength, thus allowing one to use this setup as a tool to identify the presence of $e-e$ interactions. Finally, we have studied the sign of the product of heat and charge pumped currents, showing that one can reach a situation in which the two quantities flow in the same or in opposite directions, by varying the external gate voltage.

\section{ACKNOWLEDGMENTS}

F.R., M.C., and M.S. acknowledge the support of the MIUR-FIRB2013-Project Coca (Grant No. RBFR1379UX) and the COST Action MP1209. M.C. wishes to thank A. Braggio, F. Taddei, and L. Arrachea for fruitful discussions. The authors from the Marseille group would like to acknowledge the support of Grants No. ANR-2010-BLANC-0412 ("1 shot") and No. ANR-14-CE32-0017 "one shot reloaded." This work was carried out in the framework of Labex ARCHIMEDE Grant No. ANR-11-LABX-0033 and of A*MIDEX project Grant No. ANR-11-IDEX-0001-02, funded by the "investissements d'avenir" French Government program managed by the French National Research Agency (ANR).

\section{APPENDIX A: ZERO ORDER CURRENTS}

This Appendix is devoted to the evaluation of the average currents in the absence of tunneling, taking into account the presence of the time-dependent external gate $V_{g}(x, t)$. In particular, we are interested in the average quantities in the $\mathrm{dc}$ limit or stationary regime. Here, we demonstrate that, in the absence of tunneling events (i.e., without QPCs), all average currents vanish.

Since the gate potential couples only to electrons belonging to the top edge, we do not consider here the operators related to the bottom edge. Charge and energy densities associated with the top edge [denoted with the index (1)] can be written as

$$
\begin{aligned}
& \hat{\rho}_{c}^{(1)}(x, t)=-e\left[\hat{\rho}_{R, \uparrow}(x, t)+\hat{\rho}_{L, \downarrow}(x, t)\right], \\
& \hat{\rho}_{q}^{(1)}(x, t)=v_{\mathrm{F}}\left\{\left[\hat{\rho}_{R, \uparrow}(x, t)\right]^{2}+\left[\hat{\rho}_{L, \downarrow}(x, t)\right]^{2}\right\} .
\end{aligned}
$$

The corresponding currents can be defined by using the generalized continuity equation $(v=c, q)$

$$
\partial_{t} \hat{\rho}_{v}^{(1)}(x, t)+\partial_{x} \hat{I}_{v}^{(1)}(x, t)=0 .
$$

Density operators, expressed in terms of bosonic fields, are given by

$$
\begin{gathered}
\hat{\rho}_{R, \uparrow}(x, t)=\frac{1}{2}\left[\left(\frac{1}{\sqrt{K}}+\sqrt{K}\right) \partial_{x} \hat{\phi}_{+}^{(1)}(x, t)\left(\frac{1}{\sqrt{K}}-\sqrt{K}\right) \partial_{x} \hat{\phi}_{-}^{(1)}(x, t)\right], \\
\hat{\rho}_{L, \downarrow}(x, t)=-\frac{1}{2}\left[\left(\frac{1}{\sqrt{K}}+\sqrt{K}\right) \partial_{x} \hat{\phi}_{-}^{(1)}(x, t)+\left(\frac{1}{\sqrt{K}}-\sqrt{K}\right) \partial_{x} \hat{\phi}_{+}^{(1)}(x, t)\right] .
\end{gathered}
$$


We now use the results found in Appendix B, where the time evolution of the operators $\hat{\phi}_{ \pm}^{(1)}(x, t)$ in the presence of the external gate has been calculated. Given Eqs. (B5) and (B6), we can calculate average currents in the absence of tunneling events, in the dc limit. After performing a thermal average with respect to $\hat{H}_{\mathrm{HLL}}+\hat{H}_{g}$, charge and heat currents are evaluated in the long-time limit (dc regime), using (A2),

$$
I_{c}^{(1)}(x, t)=-e K \sum_{\zeta=+,-}\left\{\theta\left(\zeta\left(x+L_{2}\right)\right) V\left(t-\zeta \frac{x+L_{2}}{u}\right)-\theta\left(\zeta\left(x-L_{1}\right)\right) V\left(t-\zeta \frac{x-L_{1}}{u}\right)\right\},
$$

for charge contribution,

$$
\begin{aligned}
I_{q}^{(1)}(x, t)= & \frac{e^{2}}{2 u}\left(K+K^{2}\right) \sum_{\zeta=+,-} \zeta\left\{\theta\left(\zeta\left(x+L_{2}\right)\right) V^{2}\left(t-\zeta \frac{x+L_{2}}{u}\right)+\theta\left(\zeta\left(x-L_{1}\right)\right)^{2}\left(t-\zeta \frac{x-L_{1}}{u}\right)\right. \\
& \left.-2 \theta\left(\zeta\left(x+L_{2}\right)\right) \theta\left(\zeta\left(x-L_{1}\right)\right) V\left(t-\zeta \frac{x+L_{2}}{u}\right) V\left(t-\zeta \frac{x-L_{1}}{u}\right)\right\}
\end{aligned}
$$

for heat current.

The expression in Eq. (A5) is a periodic function of time and it is linear in the time-dependent gate potential. Since the average over one period of $V(t)$ is zero, it is easy to realize that charge current has no dc contribution in the absence of tunneling.

On the other hand, the heat current is quadratic in the gate potential, with a nontrivial spatial dependence. In order to calculate its contribution, we thus consider two different regions with $x<-L_{2}$ and $x>L_{1}$. In the first region $(x<$ $\left.-L_{2}\right)$, the heat current, averaged over one period, reads

$$
I_{q \mathcal{L}}=-\frac{e^{2} V_{0}^{2}}{2}\left(K+K^{3}\right)\left[1-\cos \left(\omega \frac{L_{2}+L_{1}}{u}\right)\right],
$$

while in the other region $\left(x>L_{1}\right)$ one has

$$
I_{q \mathcal{R}}=\frac{e^{2} V_{0}^{2}}{2}\left(K+K^{3}\right)\left[1-\cos \left(\omega \frac{L_{2}+L_{1}}{u}\right)\right]
$$

It is worth noting that $I_{q \mathcal{R}}$ corresponds to the dc heat current flowing into the right reservoir, while $-I_{q \mathcal{L}}$ represents the dc heat current flowing into the left reservoir. Since these two contributions are equal, the net heat current globally flowing along the $2 \mathrm{D} \mathrm{TI}$ is zero. This means that the heat current introduced by the pumping mechanism, without tunneling events and backscattering, is partitioned on two equal parts, one to the left and one to the right, resulting into a zero net contribution to the pumped heat flow.

\section{APPENDIX B: PRESENCE OF AN EXTERNAL GATE}

This Appendix is devoted to the derivation of the time evolution of operators in the presence of a time-dependent external gate $V_{g}(x, t)$. As stated in the main text, we focus on a periodic time-dependent gate potential, whose explicit time dependence is

$$
V(t)=V_{0} \cos (\omega t) .
$$

We start by evaluating the time evolution of the operators $\hat{\phi}_{r, \sigma}(x, t)$ in the presence of the external gate. The Hamiltonian associated with the gate potential in Eq. (6) can be rewritten in terms of chiral bosonic fields as

$$
\hat{H}_{g}=e \sqrt{K} \int V_{g}(x, t)\left[: \partial_{x} \hat{\phi}_{+}^{(1)}(x):-: \partial_{x} \hat{\phi}_{-}^{(1)}(x):\right] .
$$

The equation of motion for the bosonic fields associated with the top edge $\hat{\phi}_{\zeta}^{(1)}(x, t)$ (in the presence of $\left.\hat{H}_{\mathrm{HLL}}+\hat{H}_{g}\right)$ is

$$
\partial_{t} \hat{\phi}_{\zeta}^{(1)}(x, t)+\zeta u \partial_{x} \hat{\phi}_{\zeta}^{(1)}(x, t)=-e \sqrt{K} V_{g}(x, t),
$$

with the corresponding solution,

$$
\begin{aligned}
\hat{\phi}_{\zeta}^{(1)}(x, t)= & \hat{\phi}_{\zeta}^{(1, \mathrm{HLL})}(x-\zeta u t) \\
& -e \sqrt{K} \int_{0}^{t} V_{g}\left[x-\zeta u\left(t-t^{\prime}\right), t^{\prime}\right] d t^{\prime} .
\end{aligned}
$$

Here, we have denoted with $\hat{\phi}_{\zeta}^{(1, \mathrm{HLL})}(x-\zeta u t)$ the solution in the absence of the external gate. Recalling the explicit form of the gate potential in Eq. (7), we have

$$
\begin{gathered}
\Delta \hat{\phi}_{+}^{(1)}(x, t)=-e \sqrt{K}\left[\theta\left(-L_{2}<x<L_{1}\right) \int_{t-\frac{L_{2}+x}{u}}^{t} d t^{\prime} V\left(t^{\prime}\right)+\theta\left(x>L_{1}\right) \int_{t-\frac{-L_{1}+x}{u}}^{t-\frac{L_{2}+x}{u}} d t^{\prime} V\left(t^{\prime}\right)\right], \\
\Delta \hat{\phi}_{-}^{(1)}(x, t)=-e \sqrt{K}\left[\theta\left(-L_{2}<x<L_{1}\right) \int_{t+\frac{-L_{1}+x}{u}}^{t} V\left(t^{\prime}\right) d t^{\prime}+\theta\left(x<-L_{2}\right) \int_{t+\frac{-L_{1}+x}{u}}^{t+\frac{L_{2}+x}{u}} V\left(t^{\prime}\right) d t^{\prime}\right],
\end{gathered}
$$

where $\Delta \hat{\phi}_{ \pm}^{(1)}(x, t)=\hat{\phi}_{ \pm}^{(1)}(x, t)-\hat{\phi}_{ \pm}^{(1, \mathrm{HLL})}(x, t)$. Recalling the explicit time dependence in Eq. (B1), the expressions in Eqs. (B5) and (B6) become

$$
\begin{aligned}
\Delta \hat{\phi}_{+}^{(1)}(x, t)= & -\frac{e V_{0}}{\omega} \sqrt{K}\left\{\theta\left(-L_{2}<x<L_{1}\right)\left[\sin (\omega t)-\sin \left[\omega\left(t-\frac{x+L_{2}}{u}\right)\right]\right]\right. \\
& \left.+\theta\left(x>L_{1}\right)\left[\sin \left[\omega\left(t-\frac{x+L_{2}}{u}\right)\right]-\sin \left[\omega\left(t-\frac{x-L_{1}}{u}\right)\right]\right]\right\},
\end{aligned}
$$




$$
\begin{aligned}
\Delta \hat{\phi}_{-}^{(1)}(x, t)= & -\frac{e V_{0}}{\omega} \sqrt{K}\left\{\theta\left(-L_{2}<x<L_{1}\right)\left[\sin (\omega t)-\sin \left[\omega\left(t+\frac{x-L_{1}}{u}\right)\right]\right]\right. \\
& \left.+\theta\left(x<-L_{2}\right)\left[\sin \left[\omega\left(t+\frac{x+L_{2}}{u}\right)\right]-\sin \left[\omega\left(t+\frac{x-L_{1}}{u}\right)\right]\right]\right\} .
\end{aligned}
$$

\section{Time evolution of fermionic operators}

We now evaluate the time evolution of fermionic operators $\hat{\psi}_{R, \uparrow}(x, t)$ and $\hat{\psi}_{L, \downarrow}(x, t)$. We recall that these operators are related to bosonic fields by the bosonization identity $[3,81]$

$$
\hat{\psi}_{R, \uparrow / L, \downarrow}(x, t) \sim e^{\frac{i}{2}\left[\left(\frac{1}{\sqrt{K}} \pm \sqrt{K}\right) \hat{\phi}_{+}^{(1)}(x, t)+\left(\frac{1}{\sqrt{K}} \mp \sqrt{K}\right) \hat{\phi}_{-}^{(1)}(x, t)\right]} .
$$

By writing $\hat{\phi}_{\zeta}^{(1)}(x, t)=\hat{\phi}_{\zeta}^{(1, \mathrm{HLL})}(x, t)+\Delta \hat{\phi}_{\zeta}^{(1)}(x, t)$, Eq. (B9) can be recast as

$$
\hat{\psi}_{R, \uparrow / L, \downarrow}(x, t)=\exp \left\{-i\left[\left(\frac{1 \pm K}{2 \sqrt{K}}\right) \Delta \hat{\phi}_{+}^{(1)}(x, t)\left(\frac{1 \mp K}{2 \sqrt{K}}\right) \Delta \hat{\phi}_{-}^{(1)}(x, t)\right]\right\}, \hat{\psi}_{R, \uparrow / L, \downarrow}^{(\mathrm{HLL})}(x, t),
$$

where we have identified with $\hat{\psi}_{R, \uparrow / L, \downarrow}^{(\mathrm{HLL})}(x, t)$ the time evolution of fermionic operator with respect to the bare $\hat{H}_{\mathrm{HLL}}$ without the external gate potential. Inserting Eqs. (B7) and (B8) into Eq. (B10) one obtains

$$
\hat{\psi}_{R, \uparrow / L, \downarrow}(x, t)=\exp \left\{i \frac{e V_{0}}{2 \omega}\left[\alpha_{\sigma}(x) \sin (\omega t)+\beta_{\sigma}(x) \cos (\omega t)\right]\right\} \hat{\psi}_{R, \uparrow / L, \downarrow}^{(\mathrm{HLL})}(x, t),
$$

in the region around the left QPC in Fig. 1 at $x \sim-d$, and

$$
\hat{\psi}_{R, \uparrow / L, \downarrow}(x, t)=\exp \left\{i \frac{e V_{0}}{\omega}(1 \pm K) \sin \left(\omega \frac{L_{2}+L_{1}}{2 u}\right) \cos \left[\omega\left(t-\frac{L_{2}-L_{1}+2 x}{2 u}\right)\right]\right\} \hat{\psi}_{R, \uparrow / L, \downarrow}^{(\mathrm{HLL})}(x, t),
$$

in the region around the right constriction located at $x=d$. In Eq. (B11) we defined the geometrical factors

$$
\begin{aligned}
& \alpha_{\uparrow / \downarrow}(x)=1-\cos \left[\omega\left(\frac{L_{2}+L_{1}}{2 u}\right)\right] \cos \left[\omega\left(\frac{x}{u}+\frac{L_{2}-L_{1}}{2 u}\right)\right] \pm K \sin \left[\omega\left(\frac{L_{2}+L_{1}}{2 u}\right)\right] \sin \left[\omega\left(\frac{x}{u}+\frac{L_{2}-L_{1}}{2 u}\right)\right], \\
& \beta_{\uparrow / \downarrow}(x)=\sin \left[\omega\left(\frac{L_{2}+L_{1}}{2 u}\right)\right] \cos \left[\omega\left(\frac{x}{u}+\frac{L_{2}-L_{1}}{2 u}\right)\right] \pm K \cos \left[\omega\left(\frac{L_{2}+L_{1}}{2 u}\right)\right] \sin \left[\omega\left(\frac{x}{u}+\frac{L_{2}-L_{1}}{2 u}\right)\right]
\end{aligned}
$$

Equations (B11) and (B12) can be expressed as

$$
\hat{\psi}_{R, \uparrow / L, \downarrow}(x, t)=e^{i A_{2 \sigma}(x) \cos \left[\omega t-\theta_{2 \sigma}(x)\right]} \hat{\psi}_{R, \uparrow / L, \downarrow}^{(\mathrm{HLL})}(x, t),
$$

in the first region around $x \sim-d$, and

$$
\hat{\psi}_{R, \uparrow / L, \downarrow}(x, t)=e^{i A_{1 \sigma} \cos \left[\omega t-\theta_{1 \sigma}(x)\right]} \hat{\psi}_{R, \uparrow / L, \downarrow}^{(\mathrm{HLL})}(x, t),
$$

in the second region around $x \sim d$. We have defined the following amplitudes and phase factors:

$$
\begin{gathered}
A_{2 \sigma}(x)=\frac{e V_{0}}{2 \omega} \sqrt{\alpha_{\sigma}^{2}(x)+\beta_{\sigma}^{2}(x)}, \\
\theta_{2 \sigma}(x)=\arctan \frac{\alpha_{\sigma}(x)}{\beta_{\sigma}(x)}+2 m \pi \quad \text { (with } m \text { integer), } \\
A_{1 \uparrow / 1 \downarrow}=\frac{e V_{0}}{\omega}(1 \pm K) \sin \left[\frac{\omega}{2 u}\left(L_{2}+L_{1}\right)\right], \\
\theta_{1 \sigma}(x)=\frac{\omega}{2 u}\left(L_{2}-L_{1}+2 x\right) .
\end{gathered}
$$

Notably it turns out that the two amplitudes $A_{1 \uparrow}$ and $A_{1 \downarrow}$ do not depend on the position $x$ in the chosen geometry.

\section{APPENDIX C: USEFUL INTEGRALS}

In the low-temperature limit $k_{\mathrm{B}} T \ll \omega_{\mathrm{c}}$ and $\omega_{\mathrm{c}} t \gg 1$, Eq. (29) becomes

$$
\mathcal{W}(t)=\ln \frac{\pi k_{\mathrm{B}} T t}{\sinh \left(\pi k_{\mathrm{B}} T t\right)\left(1+i \omega_{c} t\right)} .
$$


In this case, it is possible to perform the integral in Eq. (27) and one obtains

$$
\int d \tau \sin (n \omega \tau) P_{\gamma}\left(\tau-\frac{2 d}{u}\right) P_{\gamma}\left(\tau+\frac{2 d}{u}\right)=\frac{1}{2 i} \mathcal{H}\left(\gamma, \frac{2 d}{u} n \omega, \frac{2 d}{u} k_{\mathrm{B}} T\right)\left[\tilde{\mathcal{P}}_{2 \gamma}(n \omega)-\tilde{\mathcal{P}}_{2 \gamma}(-n \omega)\right]
$$

where

$$
\mathcal{H}(\gamma, x, y)=2 \pi \frac{\Gamma(2 \gamma)}{\Gamma(\gamma)} \frac{e^{-2 \pi \gamma y}}{\sinh \left(\frac{x}{2 y}\right)} \operatorname{Im}\left[\frac{e^{i x}}{\Gamma\left(\gamma+i \frac{x}{2 \pi y}\right) \Gamma\left(1-i \frac{x}{2 \pi y}\right)}{ }_{2} F_{1}\left(\gamma, \gamma-i \frac{x}{2 \pi y}, 1-i \frac{x}{2 \pi y} ; e^{-4 \pi y}\right)\right]
$$

and

$$
\tilde{\mathcal{P}}_{g}(\omega)=\left(\frac{2 \pi k_{B} T}{\omega_{c}}\right)^{g-1} \frac{e^{\frac{\omega}{2 k_{B} T}}}{\omega_{c}} \mathcal{B}\left[\frac{g}{2}-i \frac{\omega}{2 \pi k_{B} T}, \frac{g}{2}+i \frac{\omega}{2 \pi k_{B} T}\right]
$$

Moreover, by noticing that

$$
\begin{aligned}
& \int d \tau \sin (n \omega \tau)\left[\partial_{\tau} P_{\gamma}\left(\tau-\frac{2 d}{u}\right) P_{\gamma}\left(\tau+\frac{2 d}{u}\right)-P_{\gamma}\left(\tau-\frac{2 d}{u}\right) \partial_{\tau} P_{\gamma}\left(\tau+\frac{2 d}{u}\right)\right] \\
& =-\frac{u}{2} \partial_{d} \int d \tau \sin (n \omega \tau) P_{\gamma}\left(\tau-\frac{2 d}{u}\right) P_{\gamma}\left(\tau+\frac{2 d}{u}\right),
\end{aligned}
$$

the integral in Eq. (28) can be expressed in the following form,

$$
\begin{aligned}
& \int d \tau \sin (n \omega \tau)\left[\partial_{\tau} P_{\gamma}\left(\tau-\frac{2 d}{u}\right) P_{\gamma}\left(\tau+\frac{2 d}{u}\right)-P_{\gamma}\left(\tau-\frac{2 d}{u}\right) \partial_{\tau} P_{\gamma}\left(\tau+\frac{2 d}{u}\right)\right] \\
& =-\frac{u}{4 i} \Xi\left(\gamma, 2 d, \frac{n \omega}{u}, \frac{k_{B} T}{u}\right)\left[\tilde{P}_{2 \gamma}(n \omega)-\tilde{P}_{2 \gamma}(-n \omega)\right],
\end{aligned}
$$

where

$$
\Xi\left(\gamma, 2 d, \frac{n \omega}{u}, \frac{k_{B} T}{u}\right) \equiv \partial_{d}\left[\mathcal{H}\left(\gamma, \frac{2 d}{u} n \omega, \frac{2 d}{u} k_{B} T\right)\right]
$$

[1] S. Das Sarma and A. Pinczuk, Perspective in Quantum Hall Effects: Novel Quantum Liquid in Low-Dimensional Semiconductor Structures (Wiley, New York, 1997).

[2] G. F. Giuliani and G. Vignale, Quantum Theory of the Electron Liquid (Cambridge University Press, Cambridge, U.K., 2005).

[3] T. Giamarchi, Quantum Physics in One Dimension (Oxford University Press, Oxford, U.K., 2003).

[4] T. Martin, in Nanophysics: Coherence and Transport, edited by H. Bouchiat, Y. Gefen, S. Guéron, G. Montambaux, and J. Dalibard, Proceedings of the Les Houches Summer School of Theoretical Physics, LXXXI, 2004 (Elsevier, Amsterdam, 2005), pp. 283-359.

[5] J. P. Pekola, Nat. Phys. 11, 118 (2015).

[6] M. Büttiker, H. Thomas, and A. Prêtre, Phys. Lett. A 180, 364 (1993).

[7] E. Bocquillon, V. Freulon, F. D. Parmentier, J.-M. Berroir, B. Plaçais, C. Wahl, J. Rech, T. Jonckheere, T. Martin, C. Grenier, D. Ferraro, P. Degiovanni, and G. Féve, Ann. Phys. 526, 1 (2014).

[8] G. Fève, A. Mahé, J.-M. Berroir, T. Kontos, B. Plaçais, D. C. Glattli, A. Cavanna, B. Etienne, and Y. Jin, Science 316, 1169 (2007).

[9] F. Giazotto, P. Spathis, S. Roddaro, S. Biswas, F. Taddei, M. Governale, and L. Sorba, Nat. Phys. 7, 857 (2011).

[10] M. Carrega, D. Ferraro, A. Braggio, N. Magnoli, and M. Sassetti, Phys. Rev. Lett. 107, 146404 (2011).

[11] C. Wahl, J. Rech, T. Jonckheere, and T. Martin, Phys. Rev. Lett. 112, 046802 (2014).
[12] H. Kamata, N. Kumada, M. Hashisaka, K. Muraki, and T. Fujisawa, Nat. Nanotechnol. 9, 177 (2014).

[13] D. Ferraro, J. Rech, T. Jonckheere, and T. Martin, Phys. Rev. B 91, 205409 (2015).

[14] F. Giazotto, T. T. Heikkilä, A. Luukanen, A. M. Savin, and J. P. Pekola, Rev. Mod. Phys. 78, 217 (2006).

[15] C. Padurariu, T. Jonckheere, J. Rech, R. Melin, D. Feinberg, T. Martin, and Y. V. Nazarov, Phys. Rev. B 92, 205409 (2015).

[16] D. Ferraro, J. Rech, T. Jonckheere, and T. Martin, Phys. Rev. B 91, 075406 (2015).

[17] M. Esposito, U. Harbola, and S. Mukamel, Rev. Mod. Phys. 81, 1665 (2009).

[18] M. Campisi, P. Hänggi, and P. Talkner, Rev. Mod. Phys. 83, 771 (2011).

[19] N. Li, J. Ren, L. Wang, G. Zhang, P. Hänggi, and B. Li, Rev. Mod. Phys. 84, 1045 (2012).

[20] G. Benenti, G. Casati, T. Prosen, and K. Saito arXiv:1311.4430.

[21] R. Sánchez, B. Sothmann, and A. N. Jordan, New J. Phys. 17, 075006 (2015).

[22] R. Sanchez, B. Sothmann, and A. N. Jordan, Phys. Rev. Lett. 114, 146801 (2015).

[23] H. Thierschmann, R. Sánchez, B. Sothmann, F. Arnold, C. Heyn, W. Hansen, H. Buhmann, and L. W. Molenkamp, Nat. Nanotechnol. 10, 854 (2015).

[24] S. Jezouin, F. D. Parmentier, A. Anthore, U. Gennser, A. Cavanna, Y. Jin, and F. Pierre, Science 342, 601 (2013).

[25] M. J. Martínez-Pérez and F. Giazotto, Nat. Commun. 5, 3579 (2014). 
[26] C. Altimiras, H. le Sueur, U. Gennser, A. Anthore, A. Cavanna, D. Mailly, and F. Pierre, Phys. Rev. Lett. 109, 026803 (2012).

[27] M. Meschke, W. Guichard, and J. P. Pekola, Nature (London) 444, 187 (2006).

[28] M. F. Ludovico, J. S. Lim, M. Moskalets, L. Arrachea, and D. Sanchez, Phys. Rev. B 89, 161306 (2014).

[29] M. F. Ludovico, F. Battista, F. von Oppen, and L. Arrachea, Phys. Rev. B 93, 075136 (2016).

[30] M. Carrega, P. Solinas, M. Sassetti, and U. Weiss, Phys. Rev. Lett. 116, 240403 (2016).

[31] M. F. Ludovico, L. Arrachea, M. Moskalets, and D. Sanchez, Entropy 18, 419 (2016).

[32] M. F. Ludovico, J. S. Lim, M. Moskalets, L. Arrachea, and D. Sanchez, J. Phys.: Conf. Ser. 568, 052017 (2014).

[33] L. Arrachea and E. Fradkin, Phys. Rev. B 84, 235436 (2011).

[34] A. Calzona, M. Acciai, M. Carrega, F. Cavaliere, and M. Sassetti, Phys. Rev. B 94, 035404 (2016).

[35] M. Moskalets, Phys. Rev. Lett. 112, 206801 (2014).

[36] F. Battista, F. Haupt, and J. Splettstoesser, Phys. Rev. B 90, 085418 (2014).

[37] M. Moskalets, G. Haack, and M. Büttiker, Phys. Rev. B 87, 125429 (2013).

[38] L. Arrachea, M. Moskalets, and L. Martin-Moreno, Phys. Rev. B 75, 245420 (2007).

[39] M. Moskalets and M. Büttiker, Phys. Rev. B 66, 205320 (2002).

[40] D. J. Thouless, Phys. Rev. B 27, 6083 (1983).

[41] P. W. Brouwer, Phys. Rev. B 58, R10135 (1998).

[42] P. L. e S. Lopes, P. Ghaemi, S. Ryu, and T. L. Hughes, Phys. Rev. B 94, 235160 (2016).

[43] J. Ren, P. Hänggi, and B. Li, Phys. Rev. Lett. 104, 170601 (2010).

[44] E. C. Cuansing and J.-S. Wang, Phys. Rev. E 82, 021116 (2010).

[45] G. E. W. Bauer, E. Saitoh, and B. J. van Wees, Nat. Mater. 11, 391 (2012).

[46] G. E. W. Bauer, A. H. MacDonald, and S. Maekawa, Solid State Commun. 150, 459 (2010).

[47] S. Kolenda, M. J. Wolf, and D. Beckmann, Phys. Rev. Lett. 116, 097001 (2016).

[48] S. Murakami, N. Nagaosa, and S.-C. Zhang, Science 301, 1348 (2003).

[49] K. Uchida, S. Takahashi, K. Harii, J. Ieda, W. Koshibae, K. Ando, S. Maekawa, and E. Saitoh, Nature (London) 455, 778 (2008).

[50] J. Flipse, F. L. Bakker, A. Slachter, F. K. Dejene, and B. J. van Wees, Nat. Nanotechnol. 7, 166 (2012).

[51] F. K. Dejene, J. Flipse, G. E. W. Bauer, and B. J. van Wees, Nat. Phys. 9, 636 (2013).

[52] T. T. Heikkilä, M. Hatami, and G. E. W. Bauer, Phys. Rev. B 81, 100408 (2010).

[53] G. Dolcetto, M. Sassetti, and T. L. Schmidt, Riv. Nuovo Cimento 39, 113 (2016).

[54] X.-L. Qi and S.-C. Zhang, Rev. Mod. Phys. 83, 1057 (2011).

[55] B. A. Bernevig, T. L. Hughes, and S.-C. Zhang, Science 314, 1757 (2006).

[56] M. König, S. Wiedmann, C. Brüne, A. Roth, H. Buhmann, L. W. Molenkamp, X.-L. Qi, and S.-C. Zhang, Science 318, 766 (2007).

[57] T. L. Schmidt, Phys. Rev. Lett. 107, 096602 (2011).

[58] F. Crépin, J. C. Budich, F. Dolcini, P. Recher, and B. Trauzettel, Phys. Rev. B 86, 121106(R) (2012).

[59] A. Roth, C. Brüne, H. Buhmann, L. W. Molenkamp, J. Maciejko, X.-L. Qi, and S.-C. Zhang, Science 325, 294 (2009).
[60] I. Knez, R.-R. Du, and G. Sullivan, Phys. Rev. Lett. 107, 136603 (2011).

[61] L. Du, I. Knez, G. Sullivan, and R.-R. Du, Phys. Rev. Lett. 114, 096802 (2015).

[62] F. Reis, G. Li, L. Dudy, M. Bauernfeind, S. Glass, W. Hanke, R. Thomale, J. Schäfer, and R. Claessen, arXiv:1608.00812.

[63] R. Zhang, C. Zhang, W. Ji, P. Li, and P. Wang, arXiv:1607.03568.

[64] L. Zhou, L. Kou, Y. Sun, C. Felser, F. Hu, G. Shan, S. C. Smith, B. Yan, and T. Frauenheim, Nano Lett. 15, 7867 (2015).

[65] F. Ronetti, L. Vannucci, G. Dolcetto, M. Carrega, and M. Sassetti, Phys. Rev. B 93, 165414 (2016).

[66] T. Li, P. Wang, H. Fu, L. Du, K. A. Schreiber, X. Mu, X. Liu, G. Sullivan, G. A. Csáthy, X. Lin, and R.-R. Du, Phys. Rev. Lett. 115, 136804 (2015).

[67] A. Strom and H. Johannesson, Phys. Rev. Lett. 102, 096806 (2009).

[68] C.-Y. Hou, E.-A. Kim, and C. Chamon, Phys. Rev. Lett. 102, 076602 (2009).

[69] G. Dolcetto, S. Barbarino, D. Ferraro, N. Magnoli, and M. Sassetti, Phys. Rev. B 85, 195138 (2012); G. Dolcetto, F. Cavaliere, D. Ferraro, and M. Sassetti, ibid. 87, 085425 (2013).

[70] F. Dolcini, Phys. Rev. B 83, 165304 (2011).

[71] P. Sternativo and F. Dolcini, Phys. Rev. B 89, 035415 (2014).

[72] D. Ferraro, G. Dolcetto, R. Citro, F. Romeo, and M. Sassetti, Phys. Rev. B 87, 245419 (2013).

[73] C. de C. Chamon, D. E. Freed, S. A. Kivelson, S. L. Sondhi, and X. G. Wen, Phys. Rev. B 55, 2331 (1997).

[74] C.-W. Huang, S. T. Carr, D. Gutman, E. Shimshoni, and A. D. Mirlin, Phys. Rev. B 88, 125134 (2013).

[75] L. Vannucci, F. Ronetti, G. Dolcetto, M. Carrega, and M. Sassetti, Phys. Rev. B 92, 075446 (2015).

[76] R. Citro, F. Romeo, and N. Andrei, Phys. Rev. B 84, 161301(R) (2011).

[77] P. Virtanen and P. Recher, Phys. Rev. B 83, 115332 (2011).

[78] F. Dolcini, arXiv:1612.07575.

[79] N. Kainaris, I. V. Gornyi, S. T. Carr, and A. D. Mirlin, Phys. Rev. B 90, 075118 (2014).

[80] C. P. Orth, G. Strübi, and T. L. Schmidt, Phys. Rev. B 88, 165315 (2013).

[81] M. Sassetti and B. Kramer, Phys. Rev. Lett. 80, 1485 (1998); E. Miranda, Braz. J. Phys. 33, 3 (2003).

[82] F. Geissler, F. Crepin, and B. Trauzettel, Phys. Rev. B 92, 235108 (2015).

[83] J. I. Väyrynen and T. Ojanen, Phys. Rev. Lett. 106, 076803 (2011).

[84] T. L. Schmidt, S. Rachel, F. von Oppen, and L. I. Glazman, Phys. Rev. Lett. 108, 156402 (2012).

[85] F. Romeo, R. Citro, and M. Marinaro, Phys. Rev. B 78, 245309 (2008).

[86] P. Sharma and C. Chamon, Phys. Rev. Lett. 87, 096401 (2001).

[87] P. Sharma and C. Chamon, Phys. Rev. B 68, 035321 (2003).

[88] G. Cuniberti, M. Sassetti, and B. Kramer, Phys. Rev. B 57, 1515 (1998); M. Carrega, D. Ferraro, A. Braggio, N. Magnoli, and M. Sassetti, New J. Phys. 14, 023017 (2012).

[89] A. Braggio, D. Ferraro, M. Carrega, N. Magnoli, and M. Sassetti, New J. Phys. 14, 093032 (2012).

[90] A. Braggio, M. Sassetti, and B. Kramer, Phys. Rev. Lett. 87, 146802 (2001).

[91] M. Moskalets, Scattering Matrix Approach To Non Stationary Quantum Transport (Imperial College Press, London, 2011). 